International Journal of Applied Mathematical Research, $8(1)(2019) 12-19$
International Journal of Applied Mathematical Research
Website: www.sciencepubco.com/index.php/IJAMR
doi: $10.14419 / j$ ijamr.v8il. 24984
Research paper

\title{
Piecewise Analytic Method (PAM) is a New Step in the Evolution of Solving Nonlinear Differential Equation
}

\author{
Tamer Ahmed Abassy ${ }^{12 *}$ \\ ${ }^{1}$ Department of mathematics, Faculty of Arts and Science in Wadi Addwasir, Prince Sattam Bin Abdulaziz University, KSA. \\ ${ }^{2}$ Department of Scientific Computing,Faculty of Computers and Informatics, Benha University, Egypt. \\ *E-mail:Tamerabassy@yahoo.com
}

\begin{abstract}
In this paper, a new method is introduced for engineers and scientists which can be used for solving highly nonlinear differential equations. The method is called Piecewise Analytic Method (PAM). PAM is used to solve problems which other methods can't solve. the paper also shows how the accuracy and error can be controlled according to the needs.
\end{abstract}

Keywords: Nonlinear Differential equation; Piecewise Analytic Method; Runge-Kutta Method; Padé approximants.

\section{Introduction}

The big problem in solving the nonlinear differential equation is finding an exact analytic solution because of its difficulty and limited available functions that can be used to express the solutions analytically [1, 2, 3]. For solving this problem, it is important to find alternative forms instead of the exact analytic solution and, at the same time, the alternative forms must contain all the needed data.

During the last century, a big evolution in solving nonlinear differential equations had been achieved. The first step in this evolution was the numerical technique. The obvious advantage of the numerical methods over the analytic methods is that it often handles the nonlinear problems in simple domains. In brief, it divides the solution interval into points and finds the numerical solution at each point [4, 5].

During the last decades, another step in the evolution had been taken. This step was concerned with symbolic programming which helps to give an approximate analytic solution in the form of series solution through methods like ADM, HAM, VIM, and others [6, 7]. These methods solve the problem of finding an approximate analytic form but still have a big problem in the convergence and the limited region of convergence.

The new step in the evolution of solving a nonlinear differential equation is a combination between all of the above techniques. It helps in obtaining a general piecewise analytic solution that solves most of the problems in the previous techniques. The method is called Piecewise Analytic Method (PAM). Briefly, in Fig. 1 a diagrammatic sketch explains Piecewise Analytic Method (PAM) main steps.

\section{Piecwise Analytic Method}

Consider the $n^{\text {th }}$ order differential equation

$\frac{d^{n} u(t)}{d t^{n}}=f\left(t, u, u^{\prime}, \ldots, u^{(n-1)}\right), \quad u\left(t_{0}\right)=c_{0}, \quad u^{\prime}\left(t_{0}\right)=c_{1}, \quad \ldots \quad, u^{(n-1)}\left(t_{0}\right)=c_{(n-1)}$.

For solving (1) using PAM, three steps have to be executed as in Fig.1 .

The first step, a general approximate analytical solution $U_{m}(t)$ is obtained through rewriting equation (1) in the form

$\frac{d^{n} U_{m}(t)}{d t^{n}}=f\left(t, U_{m}, U_{m}^{\prime}, \ldots, U_{m}^{(n-1)}\right), U_{m}\left(t_{m}\right)=C_{m, 0}, \quad \frac{d U_{m}}{d t}\left(t_{m}\right)=C_{m, 1}, \quad \ldots, \frac{d^{n-1} U_{m}}{d t^{n-1}}\left(t_{m}\right)=C_{m, n-1}, \quad t \in\left[t_{m}, t_{m+1}\right], \quad m=0,1,2, \ldots$

where

$U_{m}(t)=\sum_{i=0}^{s} K_{m, i}\left(t-t_{m}\right)^{i}$ 


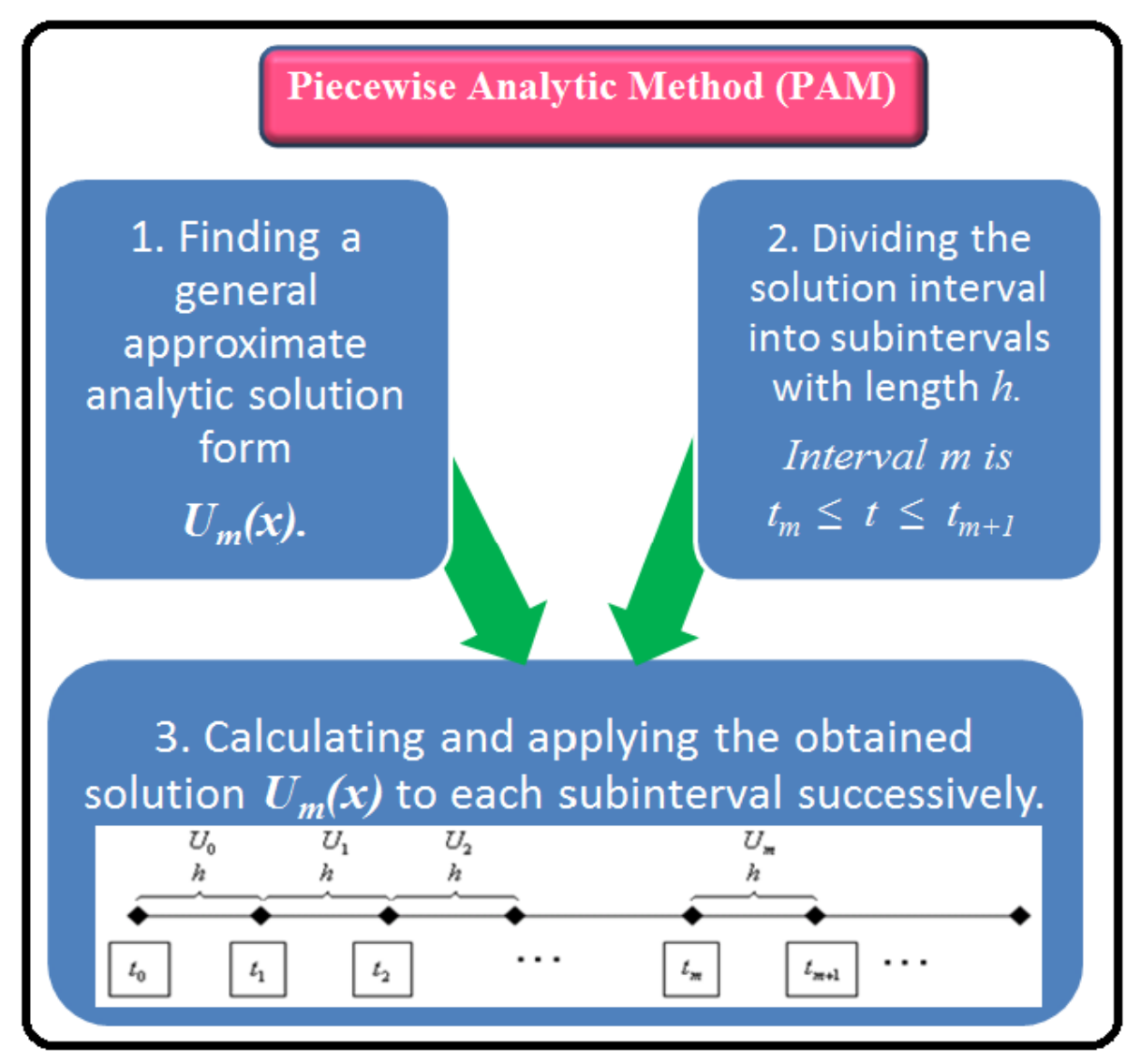

Figure 1: The main steps of PAM.

and $s$ is the order of PAM. Substituting by(3) into (2) and equating the coefficients of $\left(t-t_{m}\right)^{i}$ to zero to get a successive relations that express the coefficient $K_{m, i}$ in terms of the coefficients $K_{m, a}$ where $a<i$. At the end of this step, the general approximate analytical solution $U_{m}(t)$ is obtained and it is a function of $t, t_{m}, K_{m, 0}, K_{m, 1}, \ldots$, and $K_{m, n-1}$.

$U_{m}(t)=g\left(t, t_{m}, K_{m, 0}, K_{m, 1}, \ldots, K_{m, n-1}\right) \quad t \in\left[t_{m}, t_{m+1}\right]$

The second step is dividing the solution interval into equal subintervals, each of length $h$, and the subintervals are separated by the nodal points $t_{m}=t_{0}+m h, m=0,1,2, \ldots, n$.

The third(final) step, $U_{m}(t)$ (equation 4) is applied to each interval successively using $K_{m, 0}=C_{m, 0}=U_{m-1}\left(t_{m}\right)$ where $U_{-1}\left(t_{0}\right)=c_{0}$, $K_{m, 1}=C_{m, 1}=\frac{d U_{m-1}}{d t}\left(t_{m}\right)$ where $\frac{d U_{-1}}{d t}\left(t_{0}\right)=c_{1}, \ldots$ and $K_{m, n-1}=\frac{C_{m, n-1}}{(n-1) !}=\frac{1}{(n-1) !} \frac{d^{n-1} U_{m-1}}{d t^{n-1}}\left(t_{m}\right)$ where $\frac{d^{n-1} U_{-1}}{d t^{n-1}}\left(t_{0}\right)=c_{(n-1)}$.

In some cases, the results of the truncated Taylor series solution (3) is not accepted. For solving this problem, additional work is needed for transforming the truncated sires solution (3) into Padé approximants([14])

$U_{m}(t)=\frac{P_{l}}{Q_{k}}=\frac{\sum_{n=0}^{l} p_{m, n}\left(t-t_{m}\right)^{n}}{\sum_{n=0}^{k} q_{m, n}\left(t-t_{m}\right)^{n}}, \quad l+k=s$,

which gives excellent results. For more details see ([8]-[14]).

\section{Notes:}

1. Up to now, we have two forms for calculating( 4). The first form is the truncated Taylor series (3). The second form is Padé approximants (5).

2. The truncated Taylor series (3) is suitable if the solution has no poles and bounded, if not, the form (5) is more suitable than the truncated series (3).

3. I don't know the best form for Padé approximants (5) but by experience I prefer $l=k=$ even.

4. Dealing with the nonlinear term in (1) differs from case to another. Simply, substitute by (3) into (1), if the nonlinear term in (1) takes the polynomial form like $u^{2}, u u_{t}, u^{3}, \ldots$. If the nonlinear term in (1) takes another form like $\sin (u), e^{u}, \ldots$. In this case, it is needed to transform the nonlinear term into polynomial form first using Taylor expansion then substitute by (3) into the expansion ([11, 15]). 


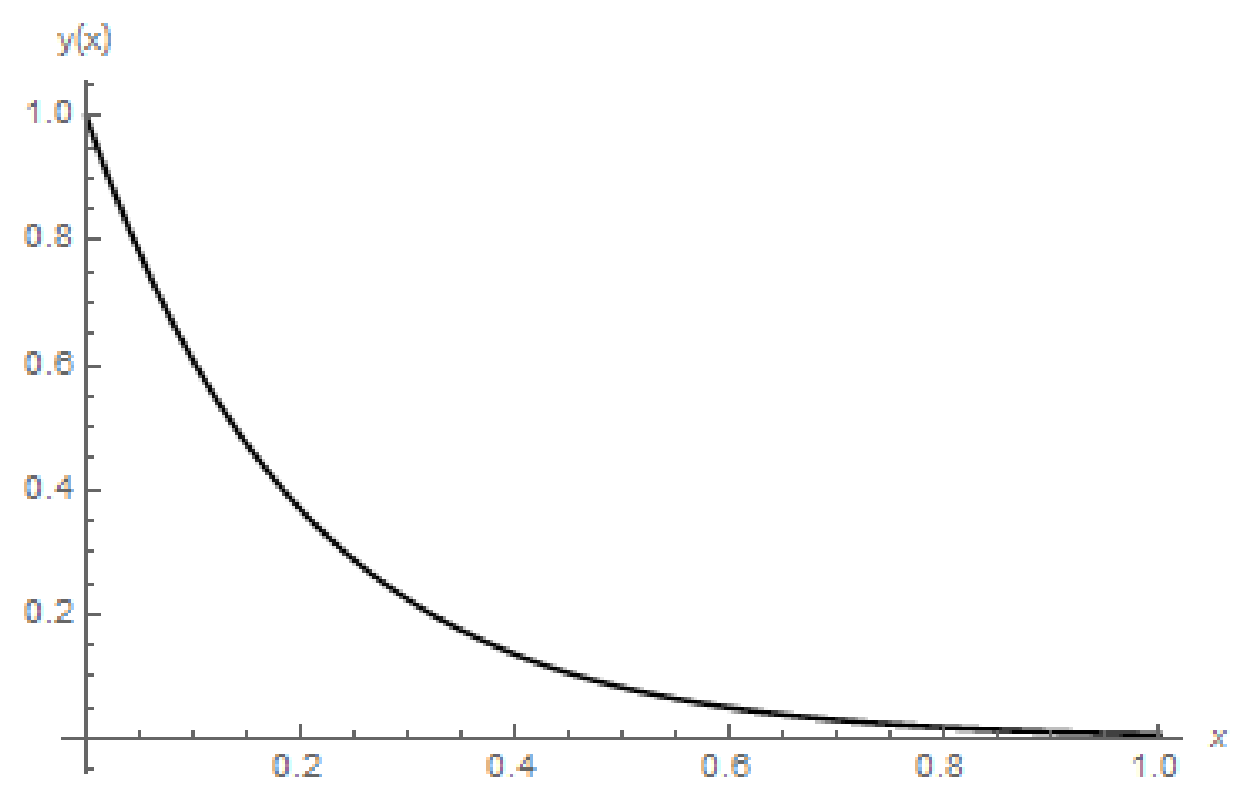

Figure 2: The exact solution $y(x)=e^{-5 x}$ using analytical method.

\section{Illustrative Examples}

\subsection{Simple Example}

Consider the differential equation

$y^{\prime}(x)=-5 y(x), \quad y(0)=1$.

This example is a simple example used to illustrate graphically the difference between analytic, numeric, series, and PAM solutions. Figs. 2-6 show graphically different forms of solution. Fig. 2 shows the exact solution

$y(x)=e^{-5 x}$.

which is the best and can be obtained through analytical methods but it is impossible to obtain for all problems. Fig. 3 shows the numerical solution using the classical Runge-Kutta method. Fig. 4 shows the series solution

$y(x) \simeq 1-5 x+\frac{25 x^{2}}{2}-\frac{125 x^{3}}{6}+\frac{625 x^{4}}{24}-\frac{625 x^{5}}{24}+\frac{3125 x^{6}}{144}-\frac{15625 x^{7}}{1008}$.

which can be obtained through one of the series methods like ADM, HPM, VIM and others. Fig. 5 shows the piecewise analytic solution(series form)

$Y_{m}(x)=f_{m}\left(1-5\left(x-x_{m}\right)+\frac{25\left(x-x_{m}\right)^{2}}{2}-\frac{125\left(x-x_{m}\right)^{3}}{6}+\frac{625\left(x-x_{m}\right)^{4}}{24}\right), \quad x_{m} \leq x \leq x_{m+1}, \quad Y_{m}\left(x_{m}\right)=f_{m}$.

Fig.6 shows another piecewise analytic solution(Padé form)

$Y_{m}(x)=\frac{\left(25\left(x-x_{m}\right)^{2}-30\left(x-x_{m}\right)+12\right) f_{m}}{25\left(x-x_{m}\right)^{2}+30\left(x-x_{m}\right)+12}, \quad x_{m} \leq x \leq x_{m+1}, \quad Y_{m}\left(x_{m}\right)=f_{m}$.

From Figs 2-6, it is clear that piecewise analytic solution Figs. 5 and 6 is better than the numerical solution Fig. 3 and the approximate analytic solution Fig. 4.

For more details about obtaining PAM solution for this example see example 1 in paper [8].

\subsection{Examples of nonlinear}

PAM can solve highly non-linear differential equation. Consider the pendulum equation which is considered as a model of nonlinearity

$m l \theta^{\prime \prime}=-m g \sin \theta-b l \theta^{\prime}+F(t)$.

which describes a simple pendulum bob of mass $m$ at the end of a weightless rod that has a fixed length $l$ at an angle $\theta$ to the vertical. $g$ is the gravity acceleration, $F(t)$ is a periodic external force pushes on the bob and $b l \theta^{\prime}$ is a friction force resistance. Equation (11) is the general second order nonlinear differential equation of simple pendulum. Studies deal with equation (11) in different forms. It is formed as linear by invoking the small angle approximation $\sin (\theta) \simeq \theta$ for small $\theta \leq 1$, nonlinear, damped $(b \neq 0)$, undamped $(b=0)$, forced $(F(t) \neq 0)$ or unforced $(F(t)=0)$. All these forms are solved using PAM in another research paper [11]. 


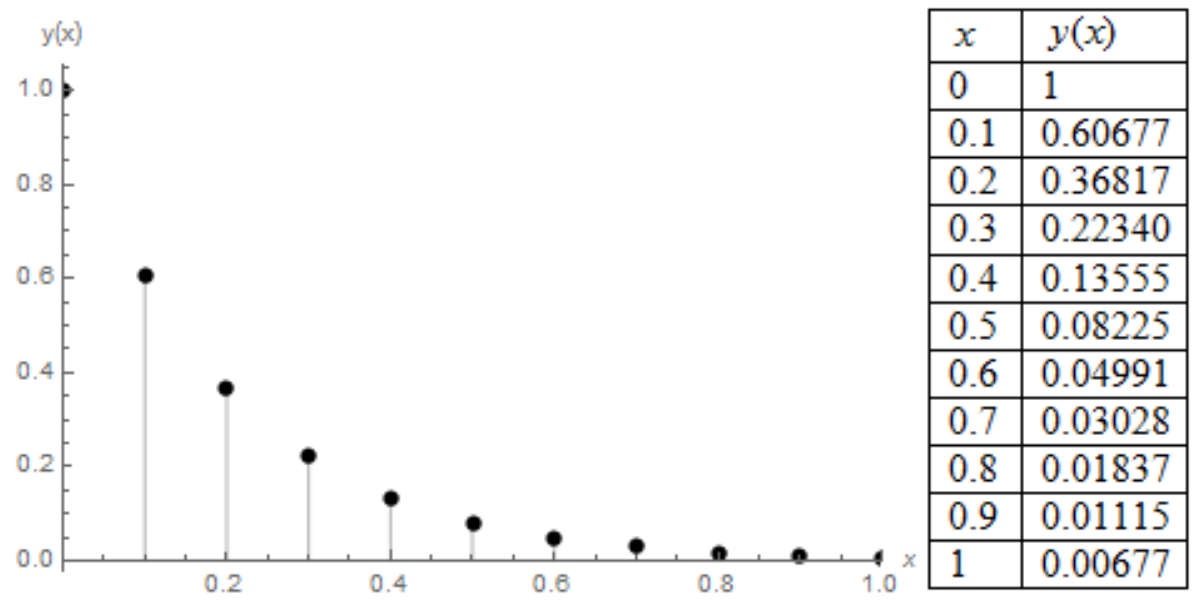

Figure 3: The numerical solution using the classical Runge Kutta method.

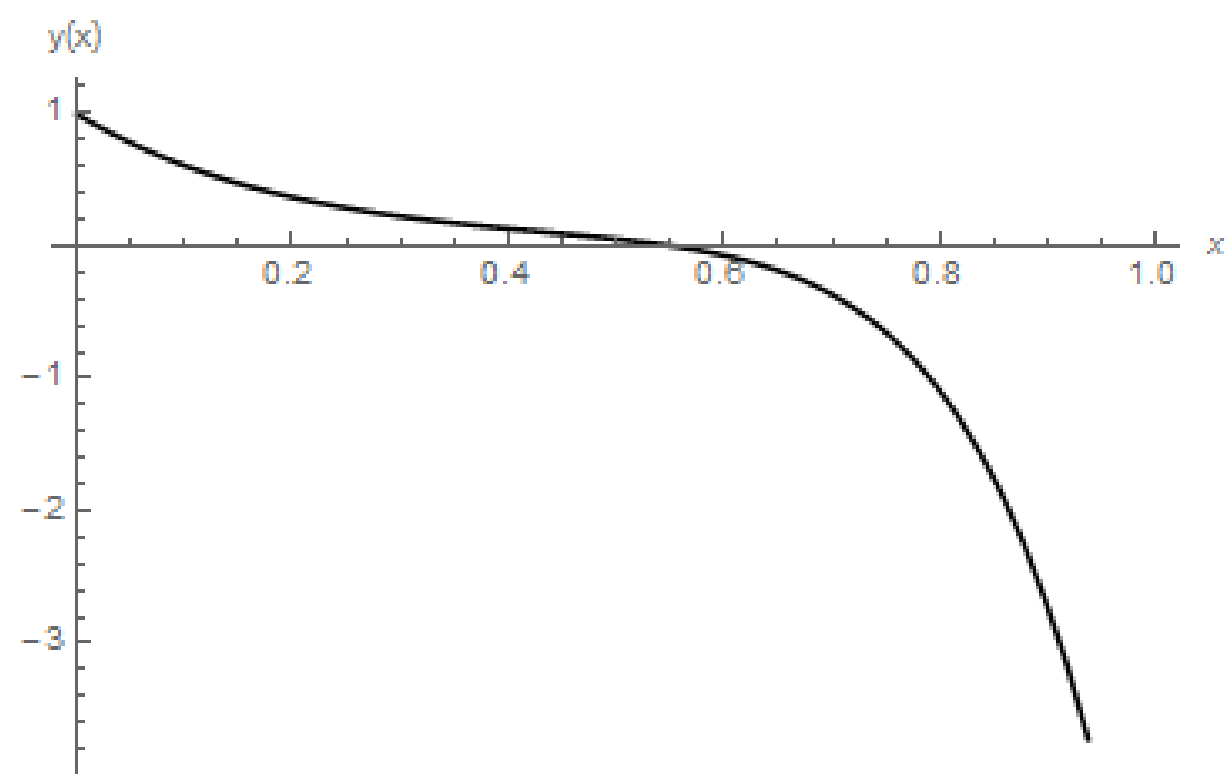

Figure 4: The series solution (8) using one of the series methods.

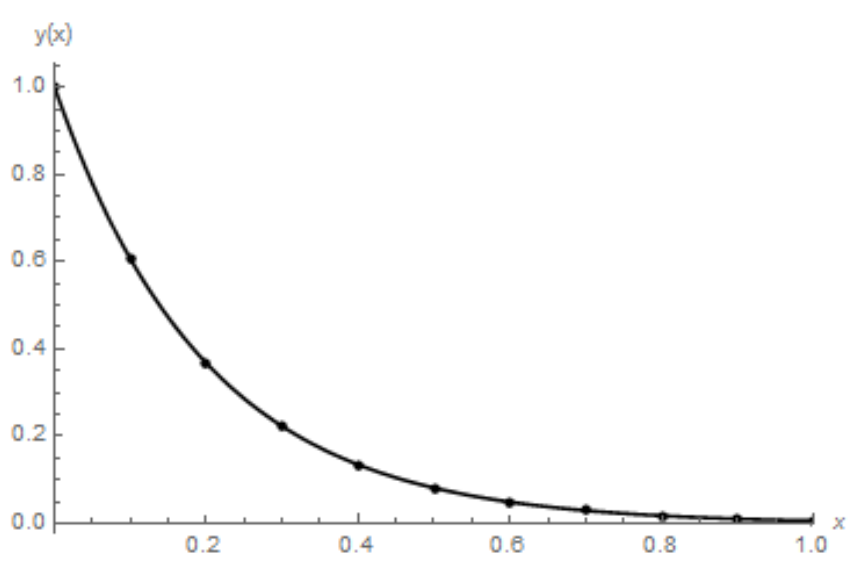

\begin{tabular}{|c|c|l|}
\hline$m$ & $x_{m}$ & $f_{m}=Y_{m-1}\left(x_{m}\right)$ \\
\hline 1 & 0 & 1 \\
\hline 2 & 0.1 & 0.60677 \\
\hline 3 & 0.2 & 0.36804 \\
\hline 4 & 0.3 & 0.22324 \\
\hline 5 & 0.4 & 0.13541 \\
\hline 6 & 0.5 & 0.08213 \\
\hline 7 & 0.6 & 0.04982 \\
\hline 8 & 0.7 & 0.03022 \\
\hline 9 & 0.8 & 0.01833 \\
\hline 10 & 0.9 & 0.01112 \\
\hline 11 & 1 & 0.00674 \\
\hline
\end{tabular}

Figure 5: The piecewise analytic solution (9). 


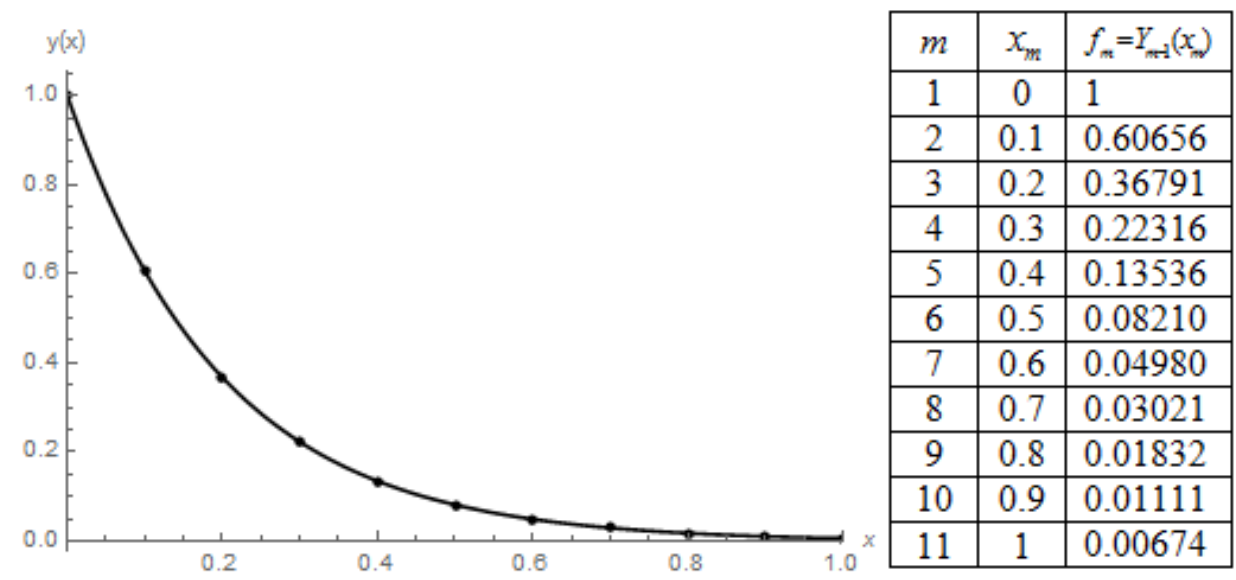

Figure 6: The piecewise analytic solution (10).

Table 1

\begin{tabular}{|c|c|c|}
\hline Index & Problem & Exact Solution $u(t)=$ \\
\hline 1 & $u^{\prime}(t)+u^{2}=0, \quad u(0)=-1$. & $\frac{1}{t-1}$ \\
\hline 2 & $u^{\prime}(t)+u^{3}=0, \quad u(0)=1$. & $\frac{1}{\sqrt{1+2 t}}$ \\
\hline 3 & $u^{\prime}(t)-u^{2}-1=0, \quad u(0)=0$. & $\tan t$ \\
\hline 4 & $u^{\prime}(t)=u-\frac{2+t}{(1+t)^{2}}, \quad u(0)=1$. & $\frac{1}{1+t}$ \\
\hline 5 & $u^{\prime}(t)-e^{t} u^{2}+u=e^{-t}, u(0)=\frac{-1}{a}$. & $-e^{-t} \frac{\cos t-a \sin t}{\sin t+a \cos t}$ \\
\hline 6 & $u^{\prime}(t)=(3-2 t) u^{2}, \quad u(0)=\frac{1}{2}$. & $\frac{1}{\left(t^{2}-3 t+2\right)}$ \\
\hline
\end{tabular}

\subsection{Examples which PAM can solve, others can't}

The numerical methods and series methods can't deal with problems which their solutions are unbounded or have poles. They give unaccepted solutions. PAM can deal with these problems. Table 1 shows some of these problems which are solved in papers $[8,9,10]$ using PAM.

\subsection{Examples which PAM gives the exact solution}

PAM gives the exact solution in two cases:

1. If the exact solution is a polynomial $\sum_{i=0}^{w} a_{i}(t)^{i}$, its order is $w$ and the truncated series (3) is used with $s \geq w$.

2. If the exact solution is a rational function $\frac{\sum_{n=0}^{z} p_{n}(t)^{n}}{\sum_{n=0}^{w} q_{n}(t)^{n}}$ and the Padé approximants (5) is used with $l \geq z$ and $k \geq w$.

The exact solution of problems 1,4 and 6 in Table 1 can be obtained using PAM [8, 9, 10].

\section{Comparing PAM with other Methods}

From previous results in this paper, published papers $[8,9,10]$ and papers $[11,16]$, it is clear PAM is better than numerical methods and other approximate methods like series methods and others.

\section{Error Estimation and PAM Control}

The accuracy of PAM solution can be controlled by two methods:

1. Changing $h$, the length of the solution interval $\left(h=t_{(m+1)}-t_{m}\right)$.

2. Changing $s$, the PAM solution order in (3).

If we take example (6), we can see the effect of changing $h$ in Table 2 and the effect of changing $s$ in Table 3.

In practical reality, If we need to solve a problem and don't know anything about the solution or need to know the effect of changing any parameters on the solution with a prescribed accuracy. How can PAM help us?

In this case, the accuracy is often estimated in a posteriori manner as follows. One calculates for specific $h$ and smaller $h$ and takes those figures which are in agreement for the two calculations. Example, if the result of using $h=0.1$ is $U=0.7447532$ while for $h=0.01$ is $U=0.744713$, then one assumes that the result $U=0.7447$ is an accurate result. For example take problem 6 , and use the notation $U_{m}^{[h, s]}(t)$ for denoting the PAM solution with step size $h$ and order of accuracy $s$. Table (4) shows the difference between two PAM solutions for two different values of $h$ and $s=4$. The same procedures can be done for increasing $s$ instead of decreasing $h$.

Note: In the limit as $s$ approaches infinity and $h$ approaches zero, PAM solution is exact, since the error bound is then zero. Of course, it does not make sense to apply a zero interval size to PAM, but the point is that we can make the error as small as we wish by selecting $s$ 
Table 2: The absolute error between the exact solution (7) and PAM solutions as $h$ changes and $s=4$.

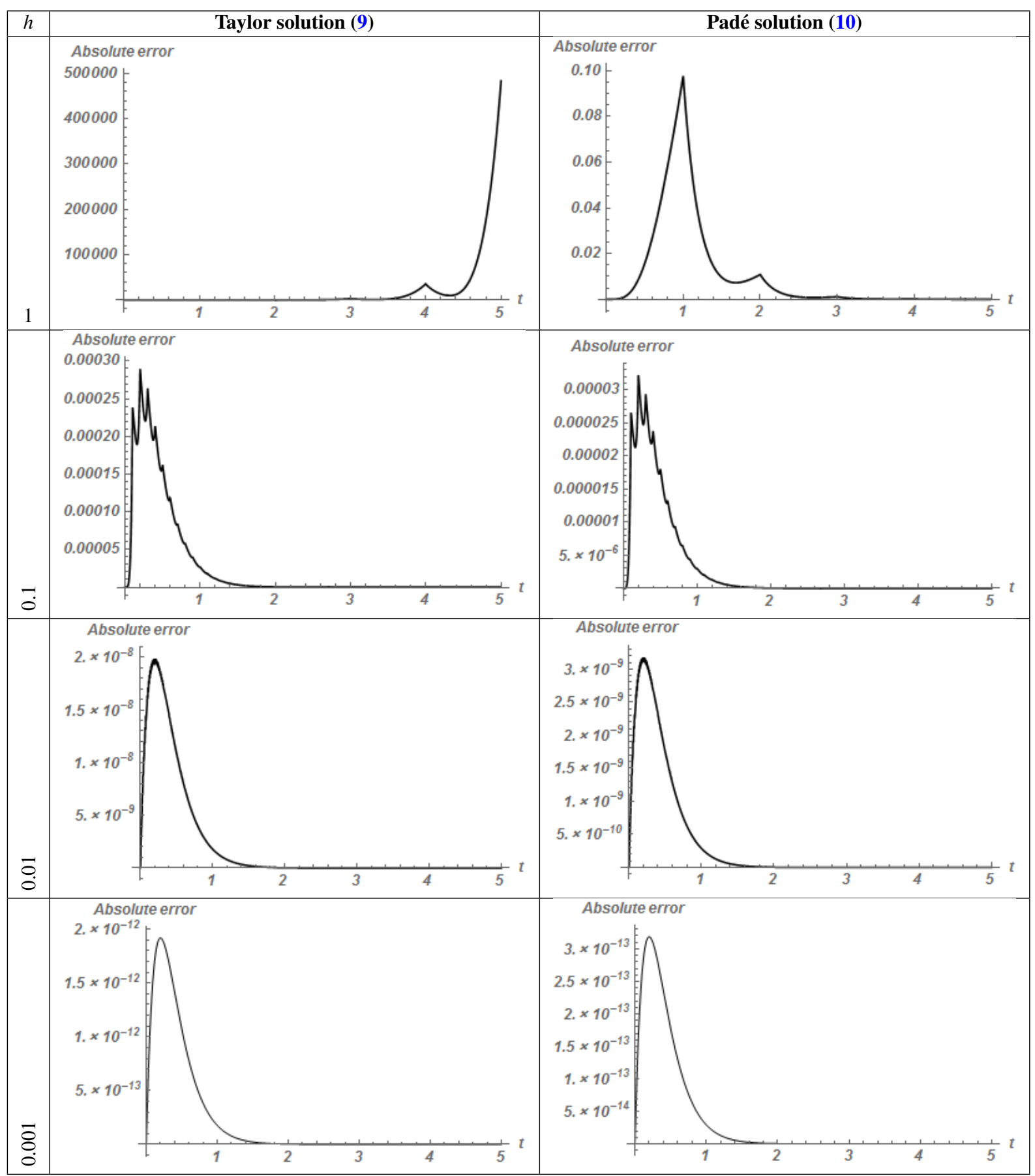


Table 3: The absolute error between the exact solution (7) and PAM solutions as $s$ changes and $h=0.1$.

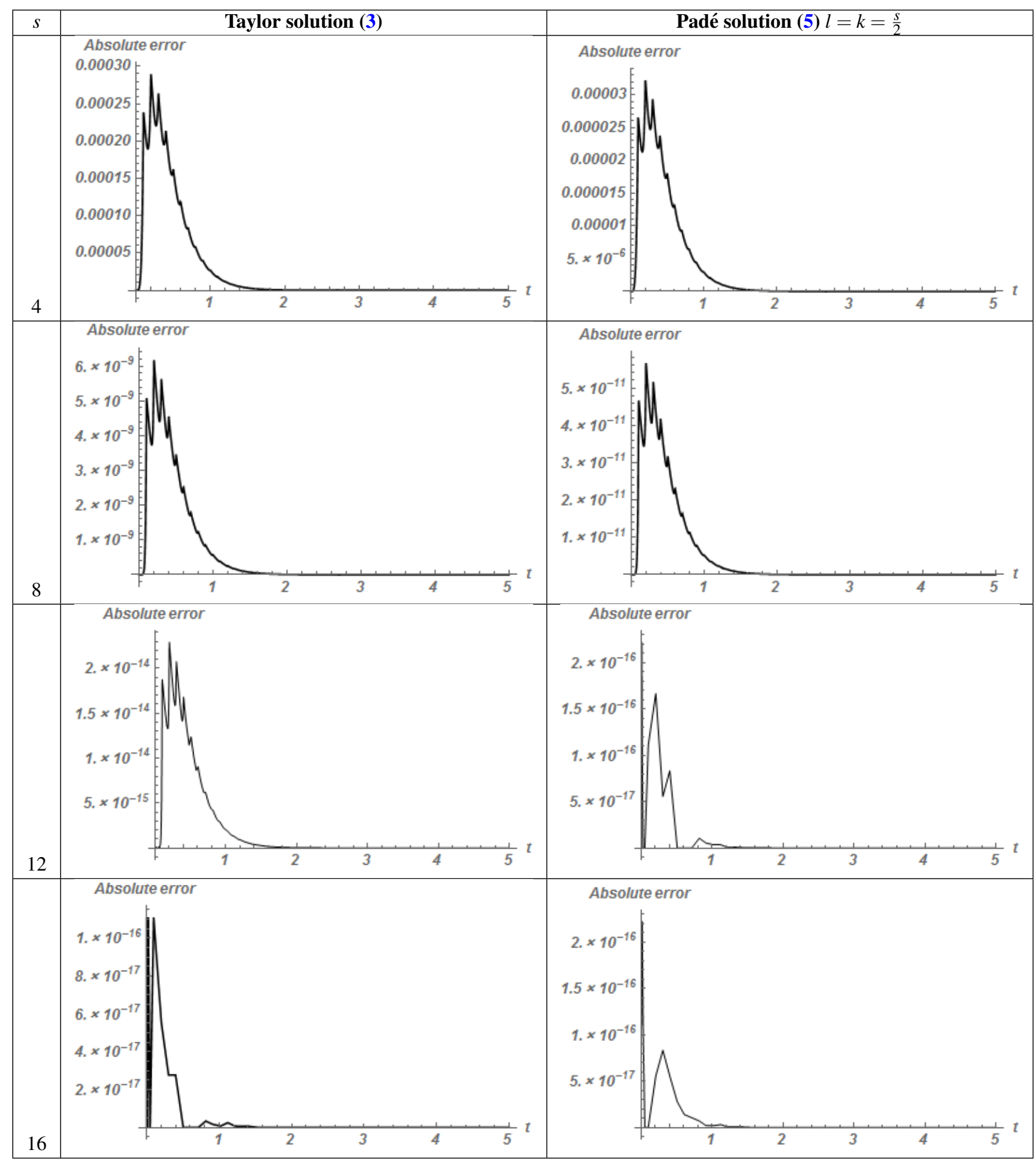


Table 4: The absolute difference between two PAM solutions as $h$ changes $s=4$.

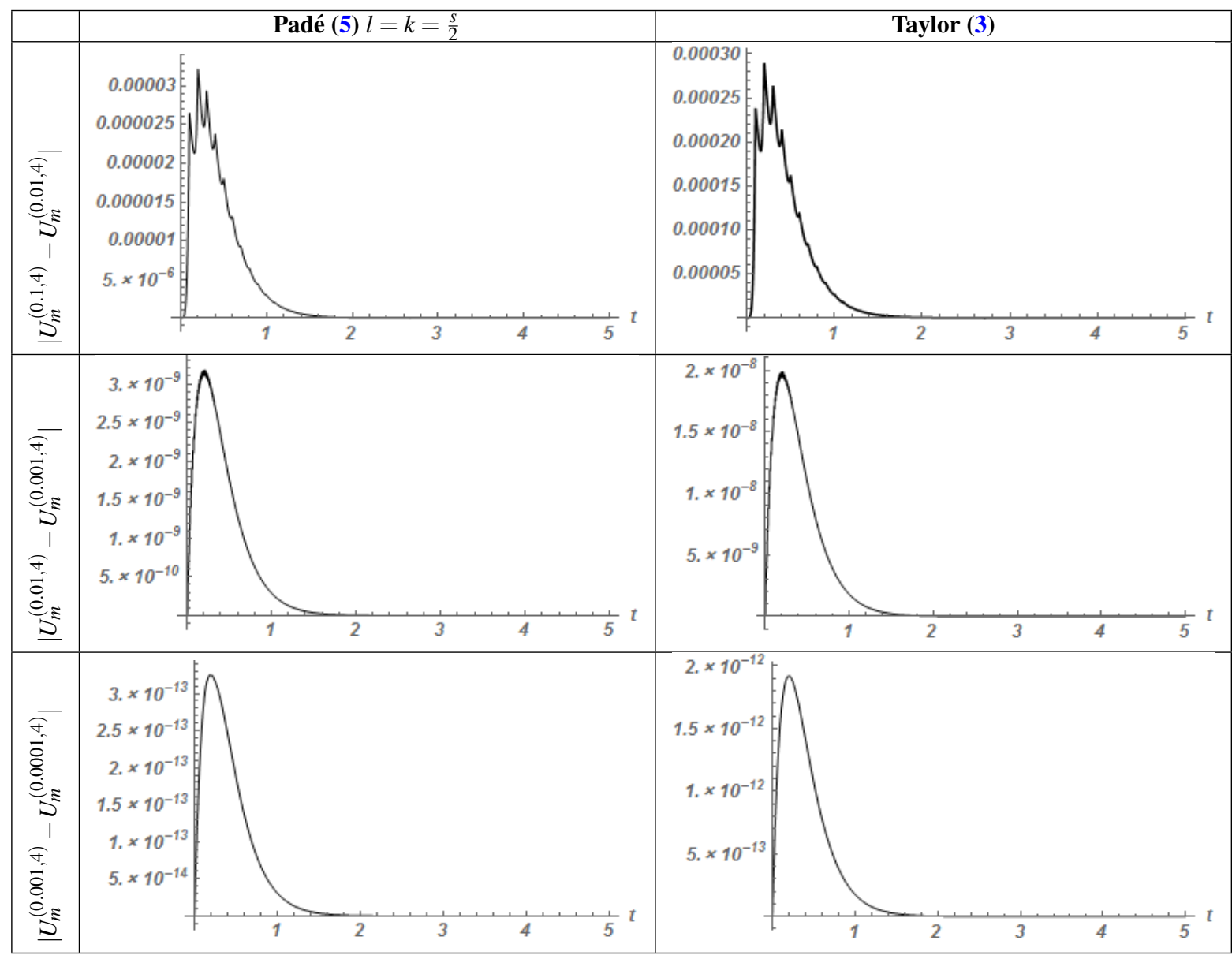

sufficiently high or $h$ sufficiently small. In this case, the truncation error in computing $U_{m}(t)$ using PAM is bounded in direct proportion with respect to the interval size $h$ and inverse proportion with respect to order of accuracy which takes us to PAM is convergent.

\section{Conclusion and contribution}

Piecewise analytic method (PAM) is a new technique used for solving nonlinear differential equation. The advantages of PAM are:

1. It gives a general analytic form that can be used in differentiation and integration.

2. It can solve highly non-linear differential equation.

3. The accuracy and error can be controlled according to our needs very easily.

4. It can solve problems which other famous techniques can't solve.

5. In some cases, it gives the exact solution.

\section{References}

[1] D. Jordan and P. Smith, Nonlinear Ordinary Differential Equations: An Introduction for Scientists and Engineers, Fourth Edition, Oxford University Press, (2007)

[2] A. H. Nayfeh and B. Balachandran, Applied non-linear dynamics, Wiley, New York (1995).

[3] D. Greenspan, Numerical Solution of Ordinary Differential Equations for Classical, Relativistic and Nano Systems. WILEY-VCH Verlag GmbH Co. $K G a A$, Weinheim, (2008).

[4] M.K. Jain, Numerical Solution of Differential Equations. 2nd Ed.,Wiley Eastern Ltd. New Delhi, (1984).

[5] J. C. Butcher, Numerical methods for ordinary differential equations, John Wiley\& SonsNew York, (2003).

[6] G. Adomian, Solving Frontier Problem of Physics: the Decomposition Method, MA: Kluwer Academic Publishers, Boston, (1994).

[7] S.J. Liao, Beyond Perturbation: Introduction to the Homotopy Analysis Method, Boca Raton: Chapman and Hall/ CRC Press, ISBN 1-58488-407-X.

[8] T. A. Abassy, Piecewise analytic method, International Journal of Applied Mathematical Research, VOL.1, NO. 1, (2012), pp. $77-107$.

[9] T. A. Abassy, Introdction to piecewise analytic method, Journal of Fractional Calculus and Applications, 3(S), (2012), pp. 1-19.

[10] T. A. Abassy, Piecewise Analytic Method (Solving Any Nonlinear Ordinary Differential Equation of 1st Order with Any Initial Condition), International Journal of Applied Mathematical Research, VOL.2, NO.1, (2013), pp.16-39.

[11] T. A. Abassy, Solving nonlinear 2nd order differential equations using piecewise analytic method (Pendulum Equations),DOI: 10.13140/RG.2.2.19063.88489/1.

[12] G. A. J. Baker, The theory and application of the padé approximant method.” In advances in theoretical physics, VOL. 1 (ed. K. A. Brueckner). New York: Academic Press (1965), pp. 1-58.

[13] G. A. J. Baker and P. Graves-Morris, Padé approximants, Cambridge University Press, New York, (1996).

[14] G. A. J. Baker, Essentials of padé approximants in theoretical physics, Academic Press, New York, (1975).

[15] Abramowitz, M. and I.A. Stegun, Handbook of Mathematical Functions with Formulas, Graphs, and Mathematical Tables, 9th printing. Dover, New York, (1972), pp. 880.

[16] T. A. Abassy, Piecewise Analytic Method VS Runge-Kutta Method (Comparative Study), DOI: 10.13140/RG.2.2.15932.90241/1. 